\title{
Acupuncture and the Future
}

\section{Opinion}

I would like to invite you to get a glimpse in the future. Specifically, in the future of the health science, let us think in medical treatment without invasive processes, without side effects, holistic, with a fast diagnosis, efficient and with a confident degree of security that presents a high percentage of success.

In the manuscript about the Projections of Global Health Outcomes from 2005 to 2060 published by World Health Organization the authors to emphasize that society need to know how the health will be like in the future, then to build a longterm to approach to setting goals to improve it [1]. There are no doubts. The human being would like to see more things happen, less obesity, drugs and alcohol abusing, best quality of life, with mental and physical health, with sports and preventive medicine for everyone.

Now I would like to introduce you to Acupuncture, which is a key component of Traditional Chinese Medicine (TCM), and acts on meridians, routes that carry vital energy (Qi) and blood (Xue), regulating Yin and Yang, connecting organs, emotions and associating the external environment with the internal environment.

The diagnosis in acupuncture also offers different features. Because of these characters above shown, the meridians conditions are identified using pulse testing, looking to the tongue aspects. It demands too much study and practice, there are abundant conditions between the acupuncture needle manipulation and pulse and tongue diagnosis, in addition to signals and symptoms.

When we talk about Traditional Chinese Medicine, we always reported to as ancient science, emerged on six thousand years working with unknown concepts. But, in truth, it isn't so. Many scientific studies had provided solid basis for further investigation of the physiological indicators of TCM constitutions in modern medicine.

In times where forecast is widely used for planning and strategic decision around industrial and economic development at organizational needs. We should look to the TCM as new possibility, which fills the gap evolving money, equipment, expertise and prevention of diseases.

Fibromyalgia is a chronical autoimmune disease, which according to the U.S. National Health Interview Survey the myocardial infarction occurred more than twice as often in patients with fibromyalgia as in patients without fibromyalgia [2]. A study in Taiwan with 58.899 patients proved that acupuncture reduced the risk, significantly, of coronary heart disease in patients with fibromyalgia [3].

Another important example of the TCM applicability is the treatment of human papilloma virus (HPV) - related warts demand a very elaborated process and there is a probably recurrence, besides this, it should be able to eliminate a large number of warts, painlessly. Through the acupoints, it has been

Oplume 9 Issue 5 - 2017
Maria Lúcia da S G Jorge*
Department of Pharmacology, Federal University of Parana,
Brazil
*Corresponding author: Maria Lúcia da SG Jorge,
Department of Pharmacology, Federal University of Parana,
IBRATE - Faculty of Technology, Brazil,
Email: mljorge@icloud.com
Received: October 30, 2017 | Published: November 28,
2017

shown that $36 \%$ of the patients treated with acupuncture had the chronic warts disappeared, and no need to work with the lesions separately [4].

Although, if the tongue and pulse are the major diagnostic methods in TCM, till now. It primordial attempts to standardize these classics methods through the original researches.

In order to examine the validity of pulse diagnosis as a modern diagnostic tool, the researchers stimulated the acupoint ST36 in healthy subjects and investigate the radial pressure pulse wave (RPPW). It was observed the increase of the peripheral pulse amplitudes, blood flow velocity (ultrasonography) and sympathetic nerve activity (HRV). The statistically significant alterations happened, including the possibility of the increased cardiac output [5].

Other way to observe the pulse changes as referee in treatment was established with patients with cervical spondylosis (CS). The study consists in observe the pulse wave before and after acupuncture treatment in these patients. The results were a pulse disorganized and irregular before the first treatment. However, the pulse became regular after treatment, indicating that acupuncture can regulate vascular function, besides effectively acting in the compression of spinal nerves and vasculature [6].

The inspection of the tongue is the other major TCM diagnostic. It involves examination of the shape, size, color, and texture of the tongue body and coat and helps reveal the state of organ functions and progression of conditions. In order to provide a clinical tongue assessment, it was study 159 patients living with HIV, who participated in an acupuncture clinical trial for chronic nausea. The aim was to explore the similarities and differences observed in tongue assessments. The overall tongue picture seen in these patients was that the tongue was swollen and tooth marked, had a pink body with cracks, and had a thick, dry white coat. The HIV disease itself and the use of long term medications affect the Blood, Qi, Yin, and Yang and the tongue diagnosis would provide a guide through into the process of the disease [7]. 
After all, another important contemporaneous disease is the Diabetes Mellitus type 2, there are an association between arterial stiffness and tongue manifestations of blood stasis in patients with type 2 diabetes, particularly a tongue with petechiae, may be associated with arterial stiffness in patients with type 2 DM. Furthermore, tongue diagnosis could detect blood stasis relevant to DM and could serve as a feasible predictor for DM [8].

May I invite you to the future?

\section{References}

1. Hughes BB, Kuhn R, Peterson CM, Rothman DS, Solorzano JR, et al. (2011) Projections of global health outcomes from 2005 to 2060 using the International Futures integrated forecasting model. Bull World Health Organ 89(7): 478-486.

2. Walitt B, Nahin RL, Katz RS, Bergman MJ, Wolfe F (2015) the prevalence and characteristics of fibromyalgia in the 2012 National Health Interview Survey. Plos One 10(9): E0138024.

3. Wu M, Huang M, Chiang J, Sun M, Lee Y, et al. (2017) Acupuncture decreased the risk of coronary heart disease in patients with fibromyalgia in Taiwan: a nationwide matched cohort study. Arthritis Res Ther 19(1): 37.
4. Brustin R, Toledano M, Geffen T, Goona R, Hochberg M, et al. (2017) Immune Modulation and Treatment of Human Papilloma VirusRelated Warts with Energetics of Living Systems Acupuncture. Med Acupunct 29(3): 145 -154.

5. Kim T, Ku B, Bae J, Shin J, Jun M, et al. (2017) Hemodynamic changes caused by acupuncture in healthy volunteers: a prospective, singlearm exploratory clinical study. BMC Complement Altern Med 17(1): 274.

6. Huiyu Z, Minfang G, Xiaozuo L (2016) Pulso changes in patients with cervical spondylosis before and after acupuncture treatment. J Tradit Chin Med February 36(1): 63-70 .

7. Anastasi JK, Chang M, Quinn J, Capili B (2014) Tongue Inspection in TCM: Observations in a Study Sample of Patients Living with HIV. Medical Acupuncture 26(1): 15-22.

8. Hsu P, Huang Y, Chiang J, Chang H, Liao P, et al. (2016) The association between arterial stiffness and tongue manifestations of blood stasis in patients with type 2 diabetes. BMC Complementary and Alternative Medicine 16: 324. 